\title{
The Equitable Total Chromatic Number of Some Join graphs
}

\author{
MA Gang \\ College of Mathematics and Computer Science \\ Northwest University for Nationalities \\ Lanzhou, Gansu, China 730030 \\ Email: jsmg@xbmu.edu.cn \\ Telephone: 13909408935
}

\author{
MA Ming \\ College of Mathematics and Computer Science \\ Northwest University for Nationalities \\ Lanzhou, Gansu, China 730030 \\ Email: jsmm@xbmu.edu.cn \\ Telephone: 13669389252
}

\begin{abstract}
A proper total-coloring of graph $G$ is said to be equitable if the number of elements (vertices and edges) in any two color classes differ by at most one, which the required minimum number of colors is called the equitable total chromatic number. In this paper, we prove some theorems on equitable total coloring and derive the equitable total chromatic numbers of $P_{m} \vee S_{n}, P_{m} \vee F_{n}$ and $P_{m} \vee W_{n}$.

Keywords-join graph; equitable total coloring; equitable total chromatic numbers
\end{abstract}

\section{INTRODUCTION}

The coloring problem is one of the most important problems in the graph theory. As an extension of proper vertex coloring, edge coloring and total coloring ${ }^{[1-5]}$, the concept and some conjectures on the equitable total coloring ${ }^{[6-8]}$ is developed. It is a very difficult problem to obtain the equitable total chromatic number, which meaningful results are rare.

The adjacent vertex distinguishing-equitable total chromatic numbers of some double graphs are research in references [9]. Zhang et al.(2008) introduced the vertex distinguishing equitable edge coloring in references [10], and the vertex distinguishing equitable edge chromatic numbers of the joingraphs between path and path, path and cycle, cycle and cycle with equivalent order are obtained in references [11].

In this paper, we study the equitable total coloring of some join graphs and get some results. Some terms and marks aren't described in this paper, please refer them to [1-3].

\section{DEFinition AND LEMmA}

Definition 2.1 ${ }^{[2]}$ For a simple graph $G(V, E)$, let $f$ be a proper $k$-edge coloring of $G$, and

$$
|| E_{i}|-| E_{j}|| \leq 1, i, j=1,2, \cdots, k .
$$

The partition $\left\{E_{i} \mid 1 \leq i \leq k\right\}$ is called a $k$-equitable edge coloring ( $k$-PEEC of $G$ in brief), and

$$
\chi_{e}^{\prime}(G)=\min \{k \mid k-\text { PEEC of } G\}
$$

is called the equitable edge chromatic number of $G$, where $\forall e \in E_{i}, f(e)=i, i=1,2, \cdots, k$.

Definition 2.2 ${ }^{[6-8]}$ For a simple graph $G(V, E)$, let $f$ be a proper $k$-total coloring of $G$, and

$$
|| T_{i}|-| T_{j}|| \leq 1, i, j=1,2, \cdots, k .
$$

The partition $\left\{T_{i}\right\}=\left\{V_{i} \cup E_{i} \mid 1 \leq i \leq k\right\}$ is called a $k$ equitable total coloring ( $k$-ETC of $G$ in brief), and

$$
\chi_{e t}(G)=\min \{k \mid k-\text { ETC of } G\}
$$

is called the equitable total chromatic number of $G$, where $\forall x \in T_{i}=V_{i} \cup E_{i}, f(x)=i, i=1,2, \cdots, k$.

Conjecture 2.1 ${ }^{[6-8]}$ For any simple graph $G(V, E)$,

$$
\chi_{e t}(G) \leq \Delta(G)+2 \text { and } \chi_{e t}(G)=\chi_{t}(G),
$$

where $\chi_{t}(G)$ is the total chromatic number of $G$.

Definition 2.3 ${ }^{[2]}$ For graph $G$ and $H(V(G) \cap V(H)=$ $\phi, E(G) \cap E(H)=\phi$ ), a new graph, denoted by $G \vee H$, is called the join of $G$ and $H$ if

$$
V(G \vee H)=V(G) \cup V(H),
$$

$E(G \vee H)=E(G) \cup E(H) \cup\{u v \mid u \in V(G), v \in V(H)\}$.

Lemma 2.1 ${ }^{[6-8]}$ For any simple graph $G(V, E)$,

$$
\chi_{e t}(G) \geq \Delta(G)+1 \text {. }
$$

Lemma 2.2 ${ }^{[2]}$ For any simple graph $G(V, E)$,

$$
\chi_{e}^{\prime}(G) \geq \Delta(G) .
$$

For any simple graph $G$ and $H, \chi_{e}^{\prime}(G)=\chi^{\prime}(G)^{[6]}$, and if $H \subseteq G$, then $\chi^{\prime}(H) \leq \chi^{\prime}(G)^{[1,2]}$, where $\chi^{\prime}(G)$ is the proper edge chromatic number of $G$. So Lemma 2.3 and Lemma 2.4 are obtained.

Lemma 2.3 For any simple graph $G$ and $H$, if $H$ is a subgraph of $G$, then

$$
\chi_{e}^{\prime}(H) \leq \chi_{e}^{\prime}(G) .
$$

Lemma 2.4 For complete graph $K_{p}$ with order $p$,

$$
\chi_{e}^{\prime}\left(K_{p}\right)= \begin{cases}p, & p \equiv 1(\bmod 2), \\ p-1, & p \equiv 0(\bmod 2) .\end{cases}
$$

Lemma $2.5^{[2,5]}$ Let $G$ be a simple graph, if $G\left[V_{\Delta}\right]$ does not contain cycle, then

$$
\chi_{e}^{\prime}(G)=\Delta(G) .
$$

Where $V\left(G\left[V_{\Delta}\right]\right)=V_{\Delta}=\{v \mid d(v)=\Delta(G), v \in$ $V(G)\}, E\left(G\left[V_{\Delta}\right]\right)=\left\{u v \mid u, v \in V_{\Delta}, u v \in E(G)\right\}$. 
Lemma 2.6 ${ }^{[6-8]}$ For complete graph $K_{p}$ with order $p$,

$$
\chi_{e t}\left(K_{p}\right)= \begin{cases}p, & p \equiv 1(\bmod 2), \\ p+1, & p \equiv 0(\bmod 2) .\end{cases}
$$

Lemma 2.7 Suppose $P_{m}$ is a Path with order $m, S_{n}, F_{n}$ and $W_{n}$ are Star, Fan and Wheel with order $n+1$, respectively. Then

$$
\Delta\left(P_{m} \vee S_{n}\right)=\Delta\left(P_{m} \vee F_{n}\right)=\Delta\left(P_{m} \vee W_{n}\right)=m+n
$$

\section{Main Results}

For some simple graphs, we obtain Theorem 3.1 and Theorem 3.2 as following.

Theorem 3.1 Let $G$ be a simple graph, if $\Delta(G)=|V(G)|$ -1 and $G$ only has a vertex with maximum degree, then

$$
\chi_{e t}(G)=\Delta(G)+1 \text {. }
$$

Proof By Lemma 2.1, we only prove that $G$ has an $f$ of $(\Delta(G)+1)$-ETC. Suppose $w \notin V(G), G^{*}=G \vee\{w\}$, then $G^{*}\left[V_{\Delta}\right]=P_{2}$, so $\chi_{e}^{\prime}\left(G^{*}\right)=\Delta\left(G^{*}\right)=\Delta(G)+1$ by Lemma 2.5.

Let $f^{*}$ be a $(\Delta(G)+1)$-PEEC of $G$,

$$
\begin{aligned}
& \forall u \in V(G), f(u)=f^{*}(w u) ; \\
& \forall u v \in E(G), f(u v)=f^{*}(u v) .
\end{aligned}
$$

Obviously, $f$ is a $(\Delta(G)+1)$-ETC of $G$, so the Theorem 3.1 is true.

Theorem 3.2 Let $G$ be a simple graph, if $\Delta(G)=|V(G)|$ -1 and $|V(G)| \equiv 1(\bmod 2)$, then

$$
\chi_{e t}(G)=\Delta(G)+1 \text {. }
$$

Proof By Lemma 2.1, we only prove that $G$ has an $f$ of $n$ ETC, where $n=|V(G)|$. Suppose $w \notin V(G), G^{*}=G \vee\{w\}$, obviously $\Delta\left(G^{*}\right)=n, G^{*} \subseteq K_{n+1}$ and $(n+1) \equiv 0(\bmod 2)$, so $\chi_{e}^{\prime}\left(G^{*}\right)=n$ by Lemma 2.2, Lemma 2.3 and Lemma 2.4.

Let $f^{*}$ be an $n$-PEEC of $G$,

$$
\begin{gathered}
\forall u \in V(G), \quad f(u)=f^{*}(w u) ; \\
\forall u v \in E(G), \quad f(u v)=f^{*}(u v) .
\end{gathered}
$$

Obviously, $f$ is an $n$-ETC of $G$, so the Theorem 3.2 is true. In the following discussion, let

$P_{m}=u_{1} u_{2} \cdots u_{m}$

$V\left(S_{n}\right)=\left\{v_{i} \mid i=0,1,2, \cdots, n\right\}, E\left(S_{n}\right)=\left\{v_{0} v_{i} \mid i=\right.$ $1,2, \cdots, n\}$;

$V\left(F_{n}\right)=\left\{v_{i} \mid i=0,1,2, \cdots, n\right\}, E\left(F_{n}\right)=\left\{v_{0} v_{i} \mid i=\right.$ $1,2, \cdots, n\} \cup\left\{v_{i} v_{i+1} \mid i=1,2, \cdots, n-1\right\}$;

$V\left(W_{n}\right)=\left\{v_{i} \mid i=0,1,2, \cdots, n\right\}, E\left(W_{n}\right)=\left\{v_{0} v_{i} \mid i=\right.$ $1,2, \cdots, n\} \cup\left\{v_{i} v_{i+1} \mid i=1,2, \cdots, n-1\right\} \cup\left\{v_{n} v_{1}\right\}$.

Theorem 3.3 When $m \geq 2$, then

$$
\chi_{e t}\left(P_{m} \vee S_{n}\right)= \begin{cases}5, & m=2, n=1, \\ m+n+1, & \text { otherwise. }\end{cases}
$$

Proof There are seven cases to be considered.

Case 1 When $m=2$ and $n=1$, obviously $P_{2} \vee S_{1}=K_{4}$. By Lemma 2.6, it's clear that the result is true.
Case 2 When $m=3, n=1$ or $m=n=2, \chi_{e t}\left(P_{3} \vee S_{1}\right)=$ $\chi_{e t}\left(P_{2} \vee S_{2}\right)=5$ by Lemma 2.7 and Theorem 3.2 , so clearly the result is true.

Case 3 When $m=2$ and $n=3$, $\chi_{\text {et }}\left(P_{3} \vee S_{3}\right) \geq 6$ by Lemma 2.1 and Lemma 2.7. We only need to prove that $P_{2} \vee S_{3}$ has an $f$ of 6-ETC. Define $f$ by

$$
\begin{aligned}
& f\left(v_{0} v_{1}\right)=f\left(v_{2} u_{1}\right)=f\left(u_{2}\right)=1 ; \\
& f\left(v_{0} v_{2}\right)=f\left(v_{3} u_{2}\right)=f\left(u_{1}\right)=2 ; \\
& f\left(v_{1} u_{2}\right)=f\left(v_{3} u_{1}\right)=f\left(v_{0}\right)=3 ; \\
& f\left(v_{0} u_{1}\right)=f\left(v_{2} u_{2}\right)=f\left(v_{3}\right)=4 ; \\
& f\left(v_{0} u_{2}\right)=f\left(v_{1} u_{1}\right)=f\left(v_{2}\right)=5 ; \\
& f\left(v_{0} v_{3}\right)=f\left(u_{1} u_{2}\right)=f\left(v_{1}\right)=6 .
\end{aligned}
$$

Obviously, the $f$ is a 6-ETC of $P_{2} \vee S_{3}$, so the result is true.

Case 4 When $m=2$ and $n \geq 4, \chi_{e t}\left(P_{2} \vee S_{n}\right) \geq n+3$ by Lemma 2.1 and Lemma 2.7. We only need to prove that $P_{2} \mathrm{~V}$ $S_{n}$ has an $f$ of $(n+3)$-ETC. Let matching $M=\left\{v_{0} v_{1}, u_{1} u_{2}\right\}$. Suppose $w \notin V\left(P_{2} \vee S_{n}\right)$, denote $G^{*}$ by

$$
V\left(G^{*}\right)=V\left(P_{2} \vee S_{n}\right) \cup\{w\},
$$$$
E\left(G^{*}\right)=E\left(P_{2} \vee S_{n} \backslash M\right) \cup\left\{w u_{1}, w u_{2}\right\} \cup\left\{w v_{i} \mid i=\right.
$$
$0,1, \cdots, n-2\}$.

Hence $G^{*}\left[V_{\Delta}\right]=u_{1} v_{0} u_{2}$ is a Path with order 3 , so $\chi_{e}^{\prime}\left(G^{*}\right)=$ $\Delta\left(G^{*}\right)=n+2$ by Lemma 2.5 .

Let $f_{1}$ be an $(n+2)$-PEEC of $G^{*}$, let $f_{2}$ be a mapping of $P_{2} \vee S_{n}$ based on $f_{1}$, that is,

$$
\begin{aligned}
& f_{2}\left(u_{i}\right)=f_{1}\left(w u_{i}\right), i=1,2 ; \\
& f_{2}\left(v_{i}\right)=f_{1}\left(w v_{i}\right), i=0,1, \cdots, n-2 .
\end{aligned}
$$

Define mapping $f_{3}$ by

$$
f_{3}\left(v_{0} v_{1}\right)=f_{3}\left(u_{1} u_{2}\right)=f_{3}\left(u_{n-1}\right)=f_{3}\left(u_{n}\right)=n+3 .
$$

Put $f=f_{1} \cup f_{2} \cup f_{3}$. So, for $P_{2} \vee S_{n}$, we

$$
\forall i \in\{1,2, \cdots, n+3\}, \quad\left|T_{i}\right|=3 \text { or } 4 .
$$

Obviously, $f$ is an $(n+3)$-ETC of $P_{2} \vee S_{n}$, hence the result is true.

Case 5 When $m \geq 4$ and $n=1, \chi_{e t}\left(P_{m} \vee S_{1}\right) \geq m+2$ by Lemma 2.1 and Lemma 2.7. We only need to prove that $P_{m} \vee$ $S_{1}$ has an $f$ of $(m+2)$-ETC. Let matching $M=\left\{v_{0} v_{1}, u_{2} u_{3}\right\}$. Suppose $w \notin V\left(P_{m} \vee S_{1}\right)$, denote $G^{*}$ by

$$
\begin{aligned}
& V\left(G^{*}\right)=V\left(P_{m} \vee S_{1}\right) \cup\{w\}, \\
& E\left(G^{*}\right)=E\left(P_{m} \vee S_{1} \backslash M\right) \cup\left\{w v_{0}, w v_{1}\right\} \cup\left\{w u_{i} \mid i=\right.
\end{aligned}
$$
$2,3, \cdots, m\}$.

Hence $G^{*}\left[V_{\Delta}\right]=v_{0} w v_{1}$ is a Path with order 3 , so $\chi_{e}^{\prime}\left(G^{*}\right)=$ $\Delta\left(G^{*}\right)=m+1$ by Lemma 2.5 .

Let $f_{1}$ be an $(m+1)$-PEEC of $G^{*}$, let $f_{2}$ be a mapping of $P_{m} \vee S_{1}$ based on $f_{1}$, that is,

$$
\begin{aligned}
& f_{2}\left(v_{i}\right)=f_{1}\left(w v_{i}\right), i=0,1 ; \\
& f_{2}\left(u_{i}\right)=f_{1}\left(w u_{i}\right), i=2,3, \cdots, m .
\end{aligned}
$$

Define mapping $f_{3}$ by

$$
f_{3}\left(v_{0} v_{1}\right)=f_{3}\left(u_{2} u_{3}\right)=f_{3}\left(u_{1}\right)=m+2 .
$$

Put $f=f_{1} \cup f_{2} \cup f_{3}$. So, for $P_{m} \vee S_{1}$, we have

$$
\forall i \in\{1,2, \cdots, m+2\}, \quad\left|T_{i}\right|= \begin{cases}3, & m=4, \\ 3 \text { or } 4, & m \geq 5 .\end{cases}
$$

Obviously, $f$ is an $(m+2)$-ETC of $P_{m} \vee S_{1}$, hence the result is true.

Case 6 When $m=3$ and $n \geq 2, \chi_{e t}\left(P_{3} \vee S_{n}\right) \geq n+4$ by Lemma 2.1 and Lemma 2.7. We only need to prove that $P_{3} \vee$ 
$S_{n}$ has an $f$ of $(n+4)$-ETC. Let matching $M=\left\{v_{0} v_{1}, v_{2} u_{2}\right\}$. Suppose $w \notin V\left(P_{3} \vee S_{n}\right)$, denote $G^{*}$ by

$$
\begin{aligned}
& V\left(G^{*}\right)=V\left(P_{3} \vee S_{n}\right) \cup\{w\}, \\
& E\left(G^{*}\right)=E\left(P_{3} \vee S_{n} \backslash M\right) \cup\left\{w u_{2}\right\} \cup\left\{w v_{i} \mid i=\right.
\end{aligned}
$$
$0,1, \cdots, n\}$.

Hence $G^{*}\left[V_{\Delta}\right]=v_{0} u_{2}$ is a Path with order 2 , so $\chi_{e}^{\prime}\left(G^{*}\right)=$ $\Delta\left(G^{*}\right)=n+3$ by Lemma 2.5 .

Let $f_{1}$ be an $(n+3)$-PEEC of $G^{*}$, let $f_{2}$ be a mapping of $P_{3} \vee S_{n}$ based on $f_{1}$, that is,

$$
\begin{aligned}
& f_{2}\left(u_{2}\right)=f_{1}\left(w u_{2}\right) \\
& f_{2}\left(v_{i}\right)=f_{1}\left(w v_{i}\right), i=0,1, \cdots, n .
\end{aligned}
$$

Define mapping $f_{3}$ by

$$
f_{3}\left(v_{0} v_{1}\right)=f_{3}\left(v_{2} u_{2}\right)=f_{3}\left(u_{1}\right)=f_{3}\left(u_{3}\right)=n+4 \text {. }
$$

Put $f=f_{1} \cup f_{2} \cup f_{3}$. So, for $P_{3} \vee S_{n}$, we have

$$
\forall i \in\{1,2, \cdots, n+4\}, \quad\left|T_{i}\right|= \begin{cases}3 \text { or } 4, & 2 \leq n \leq 6, \\ 4, & n=7 \\ 4 \text { or } 5, & n \geq 8\end{cases}
$$

Obviously, $f$ is an $(n+4)$-ETC of $P_{3} \vee S_{n}$, hence the result is true.

Case 7 When $m \geq 4$ and $n \geq 2, P_{m} \vee S_{n}$ only has a vertex $v_{0}$ with maximum degree and $d\left(v_{0}\right)=m+n=\left|V\left(P_{m} \vee S_{n}\right)\right|$ -1 , so clearly the result is true by Theorem 3.1 .

From what stated above, the proof is completed.

Theorem 3.4 When $m \geq 2$ and $n \geq 2$, then

$\chi_{e t}\left(P_{m} \vee F_{n}\right)=\left\{\begin{array}{l}7, \quad m=2, n=3 \text { or } m=3, n=2, \\ m+n+1, \quad \text { otherwise. }\end{array}\right.$

Proof Since $P_{m} \vee F_{n} \cong P_{n} \vee F_{m}$, so we only prove that the result is true when $m \geq n \geq 2$. There are six cases to be considered.

Case 1 When $m=n=2$, obviously $P_{2} \vee F_{2}=K_{5}$. By Lemma 2.6, it's clear that the result is true.

Case 2 When $m=3$ and $n=2, \chi_{\text {et }}\left(P_{3} \vee F_{2}\right) \geq 6$ by Lemma 2.1 and Lemma 2.7, obviously $P_{3} \vee F_{2}=K_{6}-u_{1} u_{3}$. Suppose $\chi_{e t}\left(K_{6}-u_{1} u_{3}\right)=6$, only the color contains at most 4 elements which colored $u_{1}$ and $u_{3}$, each color of the left contains 3 elements, so 6 colors colored at most 19 elements, but $\left|V\left(K_{6}-u_{1} u_{3}\right)\right|+\left|E\left(K_{6}-u_{1} u_{3}\right)\right|=20$. Hence, 6ETC is impossible. Moreover, 7-ETC of $P_{3} \vee F_{2}$ is getatable, denote $f$ by

$$
\begin{aligned}
& f\left(u_{i} v_{j}\right)=i+j, i=1,2,3, j=0,1,2 ; \\
& f\left(u_{1} u_{2}\right)=f\left(v_{1}\right)=5 ; f\left(u_{3}\right)=2 ; f\left(v_{0}\right)=4 \\
& f\left(u_{2} u_{3}\right)=f\left(v_{0} v_{1}\right)=f\left(v_{2}\right)=6 ; \\
& f\left(v_{0} v_{2}\right)=f\left(u_{1}\right)=7 ; f\left(v_{1} v_{2}\right)=f\left(u_{2}\right)=1 .
\end{aligned}
$$

Obviously, $f$ is a 7-ETC of $P_{3} \vee F_{2}$, hence the result is true.

Case 3 When $m \geq 4$ and $n=2, \chi_{e t}\left(P_{m} \vee F_{2}\right) \geq m+3$ by Lemma 2.1 and Lemma 2.7. We only prove that $P_{m} \vee F_{2}$ has an $f$ of $(m+3)$-ETC. Let matching $M=\left\{v_{0} u_{2}, v_{1} v_{2}\right\}$. Suppose $w \notin V\left(P_{m} \vee F_{2}\right)$, denote $G^{*}$ by

$$
V\left(G^{*}\right)=V\left(P_{m} \vee F_{2}\right) \cup\{w\},
$$

$E\left(G^{*}\right)=E\left(P_{m} \vee F_{2} \backslash M\right) \cup\left\{w v_{0}, w v_{1}, w v_{2}, w u_{2}\right\} \cup$ $\left\{w u_{i} \mid i=4,5, \cdots, m\right\}$.

Hence $G^{*}\left[V_{\Delta}\right]=v_{1} v_{0} v_{2}$ is a Path with order 3 , so $\chi_{e}^{\prime}\left(G^{*}\right)=$ $\Delta\left(G^{*}\right)=m+2$ by Lemma 2.5 .
Let $f_{1}$ be an $(m+2)$-PEEC of $G^{*}$, let $f_{2}$ be a mapping of $P_{m} \vee F_{2}$ based on $f_{1}$, that is,

$f_{2}\left(v_{i}\right)=f_{1}\left(w v_{i}\right), i=0,1,2 ; f_{2}\left(u_{2}\right)=f_{1}\left(w u_{2}\right)$; $f_{2}\left(u_{i}\right)=f_{1}\left(w u_{i}\right), i=4,5, \cdots, m$.

Define mapping $f_{3}$ by $f_{3}\left(v_{1} v_{2}\right)=f_{3}\left(v_{0} u_{2}\right)=f_{3}\left(u_{1}\right)=f_{3}\left(u_{3}\right)=m+3$.

Put $f=f_{1} \cup f_{2} \cup f_{3}$. So, for $P_{m} \vee F_{2}$, we have

$\forall i \in\{1,2, \cdots, m+3\},\left|T_{i}\right|= \begin{cases}3 \text { or } 4, & m=4,5,6, \\ 4, & m=7 \\ 4 \text { or } 5, & m \geq 8\end{cases}$

Obviously, $f$ is an $(m+3)$-ETC of $P_{m} \vee F_{2}$, hence the result is true.

Case 4 When $m=n=3, \chi_{e t}\left(P_{3} \vee F_{3}\right)=7$ by Lemma 2.7 and Theorem 3.2, so clearly the result is true.

Case 5 When $m>n=3, \chi_{\text {et }}\left(P_{m} \vee F_{3}\right) \geq m+4$ by Lemma 2.1 and Lemma 2.7. We only prove that $P_{m} \vee F_{3}$ has an $f$ of $(m+4)$-ETC. Let matching $M=\left\{v_{1} u_{2}, v_{0} v_{2}, v_{3} u_{3}\right\}$. Suppose $w \notin V\left(P_{m} \vee F_{3}\right)$, denote $G^{*}$ by

$$
\begin{aligned}
& V\left(G^{*}\right)=V\left(P_{m} \vee F_{3}\right) \cup\{w\} \\
& E\left(G^{*}\right)=E\left(P_{m} \vee F_{3} \backslash M\right) \cup\left\{w u \mid u \in V\left(P_{m} \vee\right.\right.
\end{aligned}
$$

$\left.F_{3}\right)$, and $\left.u \neq u_{1}, u_{m}\right\}$.

Hence $G^{*}\left[V_{\Delta}\right]=v_{0} v_{2}$ is a Path with order 2, so $\chi_{e}^{\prime}\left(G^{*}\right)=$ $\Delta\left(G^{*}\right)=m+3$ by Lemma 2.5 .

Let $f_{1}$ be an $(m+3)$-PEEC of $G^{*}$, let $f_{2}$ be a mapping of $P_{m} \vee F_{3}$ based on $f_{1}$, that is,

$$
f_{2}(u)=f_{1}(w u), u \in V\left(P_{m} \vee F_{3}\right) \text {, and } u \neq u_{1}, u_{m} .
$$

Define mapping $f_{3}$ by

$$
f_{3}\left(v_{1} u_{2}\right)=f_{3}\left(v_{0} v_{2}\right)=f_{3}\left(v_{3} u_{3}\right)=f_{3}\left(u_{1}\right)=f_{3}\left(u_{m}\right)=
$$
$m+4$.

Put $f=f_{1} \cup f_{2} \cup f_{3}$. So, for $P_{m} \vee F_{3}$, we have

$\forall i \in\{1,2, \cdots, m+4\},\left|T_{i}\right|= \begin{cases}4, & m=4, \\ 4 \text { or } 5, & 5 \leq m \leq 11 \\ 5, & m=12 \\ 5 \text { or } 6, & m \geq 13 .\end{cases}$

Obviously, $f$ is an $(m+4)$-ETC of $P_{m} \vee F_{3}$, hence the result is true.

Case 6 When $m \geq n \geq 4, P_{m} \vee F_{n}$ only has a vertex $v_{0}$ with maximum degree and $d\left(v_{0}\right)=m+n=\left|V\left(P_{m} \vee F_{n}\right)\right|$ -1 , so clearly the result is true by Theorem 3.1.

From what stated above, the proof is completed.

Theorem 3.5 When $m \geq 2$ and $n \geq 3$, then

$$
\chi_{\text {et }}\left(P_{m} \vee W_{n}\right)= \begin{cases}7, & m=2, n=3, \\ m+n+1, & \text { otherwise. }\end{cases}
$$

Proof There are six cases to be considered.

Case 1 When $m=2$ and $n=3$, obviously $P_{2} \vee W_{3}=K_{6}$. By Lemma 2.6, it's clear that the result is true.

Case 2 When $m=2, n=4$ or $m=n=3$, $\chi_{\text {et }}\left(P_{2} \vee\right.$ $\left.W_{4}\right)=\chi_{e t}\left(P_{3} \vee W_{3}\right)=7$ by Lemma 2.7 and Theorem 3.2, so clearly the result is true.

Case 3 When $m=2$ and $n \geq 5, \chi_{e t}\left(P_{2} \vee W_{n}\right) \geq n+3$ by Lemma 2.1 and Lemma 2.7. We only prove that $P_{2} \vee W_{n}$ has an $f$ of $(n+3)$-ETC. Define $f$ by

$$
f\left(v_{i} u_{j}\right)=i+j, i=0,1, \cdots, n, j=1,2 ;
$$




$$
\begin{aligned}
& f\left(v_{0} v_{i}\right)=i+3, i=1,2, \cdots, n ; f\left(v_{0}\right)=3 ; \\
& f\left(u_{1} u_{2}\right)=f\left(v_{1}\right)=f\left(v_{3}\right)=n+3 ; f\left(v_{1} v_{2}\right)=n+1 ; \\
& f\left(v_{2} v_{3}\right)=f\left(u_{1}\right)=n+2 ; f\left(v_{2}\right)=2 ; \\
& f\left(v_{i} v_{i+1}\right)=i-2, i=3,4, \cdots, n-1 ; \\
& f\left(v_{m} v_{1}\right)=f\left(u_{2}\right)=1 ; f\left(v_{i}\right)=i, i=4,5, \cdots, n .
\end{aligned}
$$

We have

$$
\forall i \in\{1,2, \cdots, m+3\}, \quad\left|T_{i}\right|= \begin{cases}3 \text { or } 4, & n=5 \\ 4, & n=6 \\ 4 \text { or } 5, & n \geq 7 .\end{cases}
$$

Obviously, the $f$ is an $(n+3)$-ETC of $P_{2} \vee W_{n}$, hence the result is true.

Case 4 When $m=3$ and $n \geq 4, \chi_{e t}\left(P_{3} \vee W_{n}\right) \geq n+4$ by Lemma 2.1 and Lemma 2.7. We only prove that $P_{3} \vee W_{n}$ has an $f$ of $(n+4)$-ETC. Let matching $M=\left\{v_{0} u_{2}, v_{1} v_{2}, v_{3} v_{4}\right\}$. Suppose $w \notin V\left(P_{3} \vee W_{n}\right)$, denote $G^{*}$ by

$$
V\left(G^{*}\right)=V\left(P_{3} \vee W_{n}\right) \cup\{w\}
$$$$
E\left(G^{*}\right)=E\left(P_{3} \vee W_{n} \backslash M\right) \cup\left\{w u_{2}\right\} \cup\left\{w v_{i} \mid i=\right.
$$
$0,1, \cdots, n\}$.

Hence the edge set of $G^{*}\left[V_{\Delta}\right]$ is empty, so $\chi_{e}^{\prime}\left(G^{*}\right)=$ $\Delta\left(G^{*}\right)=n+3$ by Lemma 2.5 .

Let $f_{1}$ be an $(n+3)$-PEEC of $G^{*}$, let $f_{2}$ be a mapping of $P_{3} \vee W_{n}$ based on $f_{1}$, that is,

$$
f_{2}\left(u_{2}\right)=f_{1}\left(w u_{2}\right) ; f_{2}\left(v_{i}\right)=f_{1}\left(w v_{i}\right), i=0,1, \cdots, n .
$$

Define mapping $f_{3}$ by $n+4$.

$$
f_{3}\left(v_{0} u_{2}\right)=f_{3}\left(v_{1} v_{2}\right)=f_{3}\left(v_{3} v_{4}\right)=f_{3}\left(u_{1}\right)=f_{3}\left(u_{3}\right)=
$$

Put $f=f_{1} \cup f_{2} \cup f_{3}$. So, for $P_{3} \vee W_{n}$, we have

$$
\forall i \in\{1,2, \cdots, n+4\}, \quad\left|T_{i}\right|= \begin{cases}4 \text { or } 5, & 4 \leq n \leq 10 \\ 5, & n=11 \\ 5 \text { or } 6, & n \geq 12\end{cases}
$$

Obviously, $f$ is an $(n+4)$-ETC of $P_{3} \vee W_{n}$, hence the result is true.

Case 5 When $m \geq 4$ and $n=3$, $\chi_{\text {et }}\left(P_{m} \vee W_{3}\right) \geq$ $m+4$ by Lemma 2.1 and Lemma 2.7. We only prove that $P_{m} \vee W_{3}$ has an $f$ of $(m+4)$-ETC. Let edge set $M=\left\{v_{0} v_{1}, v_{2} v_{3}, u_{2} u_{3} ; v_{0} v_{3}, v_{1} v_{2}, u_{1} u_{2}\right\}$. Suppose $w \notin$ $V\left(P_{m} \vee W_{3}\right)$, denote $G^{*}$ by

$$
\begin{aligned}
& V\left(G^{*}\right)=V\left(P_{m} \vee W_{3}\right) \cup\{w\}, \\
& E\left(G^{*}\right)=E\left(P_{m} \vee W_{3} \backslash M\right) \cup\left\{w v_{i} \mid i=0,1,2,3\right\} \cup
\end{aligned}
$$
$\left\{w u_{2}\right\} \cup\left\{w u_{i} \mid i=6,7, \cdots, m, m \geq 6\right\}$.

Hence the edge set of $G^{*}\left[V_{\Delta}\right]$ is $\left\{v_{0} v_{2}, v_{1} v_{3}\right\}, \chi_{e}^{\prime}\left(G^{*}\right)=$ $\Delta\left(G^{*}\right)=m+2$ by Lemma 2.5 .

Let $f_{1}$ be an $(m+2)$-PEEC of $G^{*}$, let $f_{2}$ be a mapping of $P_{m} \vee W_{3}$ based on $f_{1}$, that is,

$$
\begin{aligned}
& f_{2}\left(u_{2}\right)=f_{1}\left(w u_{2}\right) ; f_{2}\left(v_{i}\right)=f_{1}\left(w v_{i}\right), i=0,1,2,3 ; \\
& f_{2}\left(u_{i}\right)=f_{1}\left(w u_{i}\right), i=6,7, \cdots, m,(m \geq 6) .
\end{aligned}
$$

Define mapping $f_{3}$ by $m+3$

$$
f_{3}\left(v_{0} v_{1}\right)=f_{3}\left(v_{2} v_{3}\right)=f_{3}\left(u_{2} u_{3}\right)=f_{3}\left(u_{1}\right)=f_{3}\left(u_{4}\right)=
$$$$
f_{3}\left(v_{0} v_{3}\right)=f_{3}\left(v_{1} v_{2}\right)=f_{3}\left(u_{1} u_{2}\right)=f_{3}\left(u_{3}\right)=f_{3}\left(u_{5}\right)=
$$
$m+4$, (only if $m \geq 5$, has it vertex $u_{5}$ ).

Put $f=f_{1} \cup f_{2} \cup f_{3}$. So, for $P_{m} \vee W_{3}$, we have

$\forall i \in\{1,2, \cdots, m+4\}, \quad\left|T_{i}\right|= \begin{cases}4 \text { or } 5, & 4 \leq m \leq 10, \\ 5, & m=11, \\ 5 \text { or } 6, & m \geq 12 .\end{cases}$
Obviously, $f$ is an $(m+4)$-ETC of $P_{m} \vee W_{3}$, hence the result is true.

Case 6 When $m \geq 4$ and $n \geq 4, P_{m} \vee W_{n}$ only has a vertex $v_{0}$ with maximum degree and $d\left(v_{0}\right)=m+n=1$ $V\left(P_{m} \vee W_{n}\right) \mid-1$, so clearly the result is true by Theorem 3.1 .

From what stated above, the proof is completed.

\section{ACKNOWLEDGMENT}

This paper supported by the National Natural Science Foundation of China(61163037),the Fundamental Research Funds for the Central Universities of Northwest University for Nationalities(ZYZ2011082,ZYZ2011077).

Corresponding author: MA Ming, College of Mathematics and Computer Science, Northwest University for Nationalities, Lanzhou, Gansu, China 730030, Email: jsmm@xbmu.edu.cn

\section{REFERENCES}

[1] H. P. Yap, Total Colorings of Graphs, Berlin: Lecture Notes in Mathematics, 1623, Springer, 1996

[2] J. A. Bondy, U. S. R. Murty, Graph Theory with Applications, New York: The Macmillan Press Ltd, 1976.

[3] Tian Feng, MA Zhong-fan, Graph Theory and Network Flow Theory, Beijin: Science Press, 1987.

[4] Zhang Zhong-fu, Wang Jian-fang, The Progress of Total-Colouring of Graphs, Advances in Mathematics, 1992,21(4):390-397.

[5] Zhang Zhong-fu, Zhang Jian-xun, On Some Sufficient Conditions of First Kind Graph, Journal of Mathematics, 1985,5(2):161-165.

[6] Ma Gang, Zhang Zhong-fu, On the Equitable Total Coloring of Multiple Join-graph, Journal of Mathematical Research and Exposition, 2007,27(2):351-354.

[7] Wang Wei-fan, Equitable Total Coloring of Graphs with Maximum Degree 3, Graphs and Combin, 2002,18:677-685.

[8] Ma Gang, Zhang Zhong-fu, Qiang Hui-ying, On equitable total chromatic number of $C_{m} \bigvee F_{n}$, Journal of Lanzhou Jiaotong University, 2005,24(4): 147-149.

[9] Ma Gang, Zhang Zhong-fu, on Adjacent Vertex-distinguishing-equitable Total Coloring of Double Graphs, J. of Jilin University (Science Edition), 2009, 47(6): 1160-1164.

[10] Zhang Zhong-fu, Li Mu-chun, Yao Bin, et al, On the Vertex Distinguishing Equitable Edge-coloring of Graphs, ARS Combinatoria, 2008, 86: $193-200$.

[11] Zhang Zhong-fu, Li Jing-wen, Zhao Chuan-cheng, et al, On the Vertexdistinguishing-equitable Edge Chromatic of some Join-graphs, Acta Mathematica Sinica, Chinese Series, 2007, 50(1): 197-204. 